\title{
Patterns of failure and clinical outcomes of post-operative buccal mucosa cancers treated with adjuvant ipsilateral radiotherapy
}

\author{
Satyajeet Rath, Ajeet K. Gandhi, Madhup Rastogi, Rohini Khurana, Rahat Hadi, Harikesh B. Singh, \\ Sambit S. Nanda, Mohammad Azam, Anoop Srivastava, Avinav Bharati, Surendra Prasad Mishra \\ Department of Radiation Oncology, Dr Ram Manohar Lohia Institute of Medical Sciences, Lucknow, Uttar Pradesh, India
}

Received: July 9, 2020

Revised: August 19, 2020

Accepted: August 24, 2020

Correspondence:

Ajeet K. Gandhi

Department of Radiation Oncology, Dr Ram Manohar Lohia Institute of Medical Sciences, Lucknow, Uttar Pradesh 226010, India.

Tel: +91-40284994

E-mail: ajeetgandhi23@gmail.com ORCID:

https://orcid.org/0000-0001-5713-0963

The initial report was presented at the 2018 ASTRO Annual Meeting Abstract (No. MO_23_2678; Patterns of failure and clinical outcomes of post-operative buccal mucosa cancers treated with ipsilateral radiation therapy) in San Antonio, Texas, October 21-24, 2018 and published in the International Journal of Radiation Oncology Biology and Physics 2018;102(3 Suppl):e289.
Purpose: Adjuvant radiotherapy (RT) in buccal mucosa cancers is guided by histopathological factors. The decision to treat ipsilateral or bilateral draining lymph node is on physician discretion and guidelines do not have a defined indication regarding this. We aimed to analyze the failure patterns and survival in buccal mucosa cancers treated with adjuvant ipsilateral RT.

Materials and Methods: One hundred sixteen cases of post-operative buccal mucosa cancers-pT3 or more, node positive, close margins (1-5 $\mathrm{mm}$ ), lymphovascular invasion positive, perineural invasion positive, depth of invasion $>4 \mathrm{~mm}$-treated with RT to primary and ipsilateral nodes from May 2013 to May 2019 were retrospectively analyzed. Patients were treated to a dose of 60-66 Gy (44 Gy in the first phase and a coned down boost of 16-22 Gy in the second phase) with three-dimensional conformal radiotherapy on a linear accelerator. Primary end point was to assess control rates and secondary end point was to evaluate the overall survival (OS) and disease-free survival (DFS) outcomes.

Results: Median age was 46 years with male; female ratio of 110:6. The edition of the American Joint Committee on Cancer stage distributions were I (3.4\%), II (34.4\%), III (24.1\%), and IV (37.9\%). At a median follow-up of 22 months, crude rates of local failure, regional failure, and contralateral neck failure were $9.4 \%, 10.3 \%$, and $3.4 \%$, respectively. The 2 -year contralateral neck control rate was $94.9 \%$. Pathological positive node portended poorer OS (86.6\% vs. $68.6 \% ; p=0.015)$ and DFS $(86.5 \%$ vs. $74.9 \% ; p=0.01)$.

Conclusion: Incidence of contralateral recurrence with ipsilateral irradiation in buccal mucosa cancers is low with descent survival outcomes, particularly in node negative cases.

Keywords: Adjuvant radiotherapy, Carcinoma, Mouth mucosa

\section{Introduction}

Oral cavity cancers account for approximately 0.35 million new cases and 0.18 million deaths annually, worldwide [1]. India accounts for roughly one-third of the new cases and around half the number of deaths [1]. Buccal mucosa cancers accounts for around half of these cancers $[2,3]$. Standard therapy for buccal mucosa cancers is optimal surgical resection followed by adjuvant treatment [4]. Adjuvant radiotherapy after surgery is indicated in cases with pathological tumor size of pT3 or more, pathological node

Copyright (C) 2020 The Korean Society for Radiation Oncology

This is an Open Access article distributed under the terms of the Creative Commons Attribution Non-Commercial License (http://creativecommons.org/licenses/by-nc/4.0/) which permits unrestricted non-commercial use, distribution, and reproduction in any medium, provided the original work is properly cited. 
positivity, close margins, lymphovascular invasion (LVI) positive, perineural invasion (PNI) positive, depth of invasion (DOI) $>6 \mathrm{~mm}$ [5]. Around $60 \%$ cases of buccal cancers present with advanced stage and patients tend to have multiple risk factors [6,7]. Post-operative radiotherapy with or without chemotherapy is the standard of care after resection of locally advanced carcinomas, as these patients are higher risk for locoregional relapse [8]. The volumes for adjuvant radiotherapy generally include the primary tumor bed, ipsilateral and or contralateral nodal volumes. Standard guidelines regarding the inclusion or exclusion of contralateral draining lymph nodes as elective volume in adjuvant radiotherapy does not exist and are mostly guided by clinician discretion. For patients with clear margins and no extracapsular extension, elective contralateral neck radiation is suggested for cases with high risk like advanced disease approaching midline and multiple positive nodes in the ipsilateral neck which increase the risk of nodal recurrence in the contralateral neck [9]. However, contralateral neck irradiation is not routinely performed [9-11]. Various clinical studies opine that ipsilateral neck dissection results in good outcomes with very limited contralateral neck failure in buccal mucosa cancers $[12,13]$ setting the pretext for ipsilateral adjuvant radiotherapy in selected subset of buccal mucosa cancers.

There are no detailed guidelines regarding the management of buccal cancers in terms of ipsilateral or bilateral neck irradiation. However, selected subset of buccal mucosa cancers presenting with limited number of risk factors and favourable prognosis can be treated with ipsilateral radiotherapy to neck nodes and tumor bed, thereby limiting toxicity but without impairing outcomes. Literature on patterns of failure in these group of patients is sparse. The general practice followed at our institute is to irradiate the ipsilateral face and neck in patients intermediate risk disease [14]. We therefore conducted a retrospective analysis of prognostic factors, failure patterns and survival outcomes in these cohort of buccal cancers treated with ipsilateral irradiation at our institute and tried to identify subgroup(s) with poor prognosis suitable for more aggressive therapeutic approach.

\section{Methods and Materials}

\section{Patients}

It was a retrospective analysis of buccal mucosa squamous cell carcinoma patients, treated with surgery and radiotherapy to primary and ipsilateral nodes from May 2013 to May 2019. The data of 1,400 patients of head and neck cancer treated with radiotherapy was retrieved from the departmental archives of radiation oncology department. From this data, 116 cases of buccal mucosa cancers who underwent curative resection and received irradiation to primary tumor and ipsilateral neck were identified. The consort diagram representing the actual number of patient data retrieved has been mentioned in Fig. 1. All the records were reviewed for each patient: age, gender, site of primary tumor, stage as per the 7th edition of the American Joint Committee on Cancer [15], histological findings after surgery, local and regional recurrence in ipsilateral and contralateral neck, disease-free survival (DFS) and overall survival (OS). The patients receiving neoadjuvant chemotherapy were excluded. The patients having tumor in any site other than buccal mucosa and multiple oral cavity and/or head and neck cancers or previously treated oral cancers were also excluded from the study.

\section{Treatment}

All patients underwent adequate resection of the primary tumor and neck nodes. Surgery of the primary tumor consisted of wide local excision or composite resection with/without marginal or segmental mandibulectomy depending upon the extent of the disease. Neck dissection included either supra-omohyoid or modified radical neck dissection. Patients with any or more of the following risk factors were included in the study: pathological tumor size of pT3 or more, pathological node positivity, close margins, LVI positive, PNI positive, $\mathrm{DOI}>6 \mathrm{~mm}$. While evaluating various outcomes in terms of $\mathrm{DOI},<12 \mathrm{~mm}$ and $>12 \mathrm{~mm}$ were use as parameters [13]. All patients had lateralized primary tumors with adequate surgical resection.

Clinical target volumes (CTV) consisted of the post-operative tumor bed with a margin of $1 \mathrm{~cm}$ and the ipsilateral nodal volumes. The levels of the nodal volumes were decided as per the risk factors present in the post-operative histopathological factors and the possible sites of clinical and nodal spread. The planning target vol-

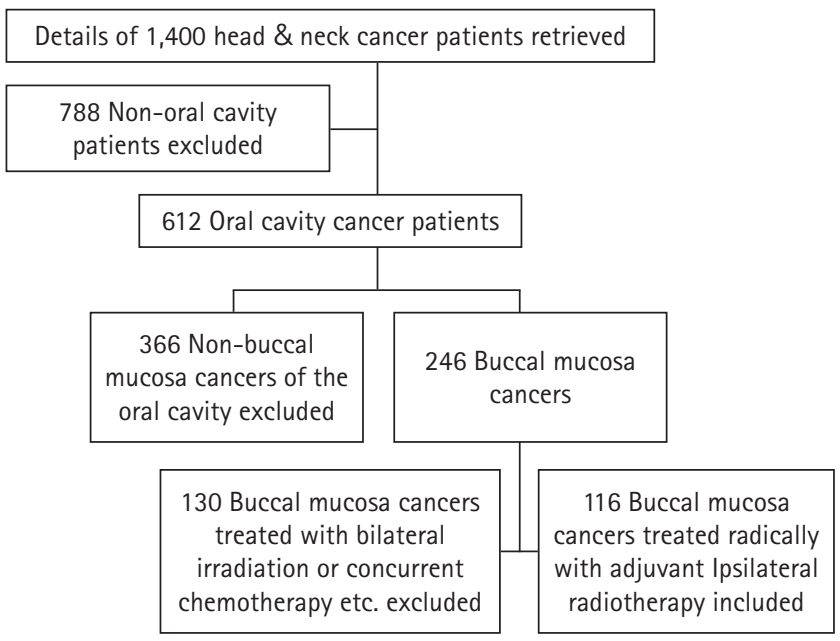

Fig. 1. Consort diagram depicting the selection of patients for the present analysis. 
ume (PTV) included CTV with a 0.5-cm uniform margin to account for set up and motion errors. Radiotherapy was delivered using 6-MV photons to a dose of 60-66 Gy, using a 2-Gy dose fractionation, one fraction per day, 5 days per week by using linear accelerator (Infinity and Synergy; Elekta, Crawley, UK) with a collimator leaf width of $1 \mathrm{~cm}$ at the isocentre. Patients were treated with three-dimensional conformal radiotherapy using either parallel opposed anterior-posterior oblique fields in a single phase to a dose of 60-66 Gy respecting the spinal cord tolerance or using anterior and lateral field in two phases, in which 44 Gy was delivered in the first phase and a coned down boost of 16-22 Gy was delivered in the second phase. The median dose of radiotherapy was $60 \mathrm{~Gy}$ (range, 58 to $66 \mathrm{~Gy}$ ). All patients were followed up on a regular basis: monthly once for first 6 months, every 2 months for the next year, every 3 months for the third and fourth years, and then 6 months to annually, thereafter.

\section{Statistics}

Primary end point was to assess local and regional control rates and secondary end point was to evaluate the survival outcomes: OS, DFS, and distant metastasis-free survival (DMFS). Locoregional failure was defined as the appearance of tumor in the post-operative bed or cervical node metastasis or both. All time intervals were calculated from the date of registration in the radiation oncology or surgical oncology department (whichever was earlier) to the date of event of interest. OS was measured from the date of registration to the date of death from any cause. DFS was defined as the time from the day of registration to date of failure (either locoregional or distant or both) or death. DMFS was defined as the time interval until the development of distant metastasis. Statistical analysis was performed with SPSS statistical software package for Mac (version 23.0; IBM, Armonk, NY, USA). All survival analyses were performed using Kaplan-Meier method. Log-rank test was used to test the statistical significance of differences in the survival and control rates. $p<0.05$ was considered statistically significant. All potential prognostic factors were analyzed. Cox regression analysis was used to perform multivariate analysis on factors found significantly associated with outcomes in univariate analysis.

\section{Results}

\section{Clinicopathological factors}

Median age was 46 years (range, 28 to 80 years) with male; female ratio of 110:6. All patients underwent ipsilateral neck dissection, except three patients who underwent bilateral neck dissection. Stage of the patients in terms of pathological tumor size, patho- logical nodal size and overall pathological staging have been mentioned in Table 1. Four patients of stage I (pT1N0) were taken up for adjuvant radiotherapy as they had one or more high-risk factors for recurrence (two of them had PNI, one had lymphovascular space invasion and three patients had DOI more than $10 \mathrm{~mm}$ ). Fifty-six patients (48.27\%) had clinically positive nodes, but 48/116

Table 1. Clinicopathological factors $(n=116)$

\begin{tabular}{|c|c|}
\hline Characteristic & Number of patients (\%) \\
\hline \multicolumn{2}{|l|}{ Age (yr) } \\
\hline$<50$ & $64(55)$ \\
\hline$\geq 50$ & $52(45)$ \\
\hline \multicolumn{2}{|l|}{ Sex } \\
\hline Male & 110 (95) \\
\hline Female & $6(5)$ \\
\hline Tobacco addiction & $102(87.9)$ \\
\hline Alcohol abuse & $69(59.4)$ \\
\hline \multicolumn{2}{|l|}{ pT stage } \\
\hline 1 & $11(9.5)$ \\
\hline 2 & $63(54.3)$ \\
\hline 3 & $20(17.2)$ \\
\hline 4 & $22(19.0)$ \\
\hline \multicolumn{2}{|l|}{ pN stage } \\
\hline 0 & $68(58.6)$ \\
\hline 1 & 37 (31.9) \\
\hline 2 & $11(9.5)$ \\
\hline \multicolumn{2}{|l|}{ Overall TNM stage } \\
\hline I & $4(3.4)$ \\
\hline$\|$ & $40(34.4)$ \\
\hline III & $28(24.1)$ \\
\hline IV & $44(37.9)$ \\
\hline \multicolumn{2}{|l|}{ Grading } \\
\hline WD & $59(50.9)$ \\
\hline MD & $51(43.9)$ \\
\hline PD & $6(5.2)$ \\
\hline \multicolumn{2}{|l|}{$\mathrm{DOI}(\mathrm{mm})$} \\
\hline$<12$ & $63(54.3)$ \\
\hline$\geq 12$ & $53(45.7)$ \\
\hline \multicolumn{2}{|c|}{ Risk factors for PORT } \\
\hline pT3 or more & $42(36.2)$ \\
\hline $\mathrm{pN}+$ & $48(41.4)$ \\
\hline PNI & $31(26.7)$ \\
\hline LVI & $34(29.3)$ \\
\hline DOI & $46(39.7)$ \\
\hline
\end{tabular}

pT, pathological tumor stage, $\mathrm{pN}$, pathological nodal stage; WD, well differentiated; $M D$, moderately differentiated; $P D$, poorly differentiated; $\mathrm{PNI}$, perineural invasion; LVI, Iymphovascular invasion; DOI, depth of invasion; PORT, post-operative radiotherapy.

${ }^{a}$ The total may not add up to $100 \%$ due to multiple factors present in each patient. 
(41.3\%) were pathologically positive. The ipsilateral neck was irradiated in 113 cases (97.4\%), whereas 3 cases received radiotherapy only to the primary tumor bed.

\section{Locoregional control, local control, and regional control}

Median follow-up was 22 months (range, 6 to 163 months). The 2-year locoregional control (LRC), local control (LC), and regional control (RC) rates were $80.9 \%, 88.4 \%$, and $89.5 \%$, respectively. There were 23 locoregional failures. The crude $\mathrm{LC}$ and $\mathrm{RC}$ rate were $90.5 \%$ and $89.7 \%$, respectively. There was no significant difference in LRC, LC, and RC with clinicopathologic factors like age, sex, pT stage, overall tumor stage, LVI, PNI, DOI or grade (Table 2). There was statistically significant difference in 2-year LRC rates for different nodal stages (pN0 vs. pN1 vs. pN2: $86.1 \%$ vs. $71.5 \%$ vs. $77.9 \% ; p=0.036$ ) (Fig. 2). The factor significantly influencing LRC rate ( $p N 0$ vs. $\mathrm{pN} 1+2: 86.1 \%$ vs. $72.9 \% ; \mathrm{p}=0.016$ ) and $\mathrm{RC}$ rate (pNo vs. $\mathrm{pN} 1+2: 100 \%$ vs. $81.8 \% ; \mathrm{p}=0.037$ ) was pathological nodal staging, although it did not influence the LC rates.

\section{Overall survival}

The 2-year OS for the entire cohort was 79.5\%. The number of

Table 2. Variation of outcomes with prognostic factors in univariate analysis

\begin{tabular}{|c|c|c|c|c|c|}
\hline \multirow{2}{*}{ Prognostic factor analyzed } & \multicolumn{5}{|c|}{$p$-value } \\
\hline & OS & DFS & $\mathrm{LRC}$ & LC & $\mathrm{RC}$ \\
\hline pT (1 vs. 2 vs. 3 vs. 4 ) & 0.500 & 0.635 & 0.796 & 0.176 & 0.642 \\
\hline pN (0 vs. 1 vs. 2) & $0.045^{*}$ & $0.032^{*}$ & $0.036^{*}$ & 0.185 & $0.031^{*}$ \\
\hline pN (0 vs. 1/2) & $0.015^{*}$ & $0.010^{*}$ & $0.016^{*}$ & 0.136 & $0.037^{*}$ \\
\hline pTNM (1 vs. 2 vs. 3 vs. 4) & 0.914 & 0.897 & 0.897 & 0.314 & 0.659 \\
\hline pTNM (1/2 vs. 3/4) & 0.554 & 0.653 & 0.563 & 0.987 & 0.470 \\
\hline LVI (negative vs. positive) & 0.064 & 0.159 & 0.268 & 0.062 & 0.911 \\
\hline PNI (negative vs. positive) & 0.479 & 0.593 & 0.356 & 0.948 & 0.321 \\
\hline Grade ( 1 vs. 2 vs. 3 ) & 0.804 & 0.808 & 0.683 & 0.132 & 0.927 \\
\hline Grade (1+2 vs. 3$)$ & 0.653 & 0.712 & 0.627 & 0.203 & 0.553 \\
\hline $\mathrm{DOI}$ (<12 vs. $\geq 12 \mathrm{~mm}$ & 0.869 & 0.925 & 0.944 & 0.156 & 0.348 \\
\hline Sex (male vs. female) & 0.846 & 0.766 & 0.173 & 0.415 & 0.319 \\
\hline Age (<50 vs. $\geq 50 \mathrm{yr})$ & 0.453 & 0.371 & 0.582 & 0.162 & 0.542 \\
\hline
\end{tabular}

OS, overall survival; DFS, disease-free survival; LRC, locoregional control; LC, local control; RC, regional control; pT, pathological tumor stage, pN, pathological nodal stage; LVI, lymphovascular invasion; PNI, perineural invasion; DOI, depth of invasion.

${ }^{*} p<0.05$.
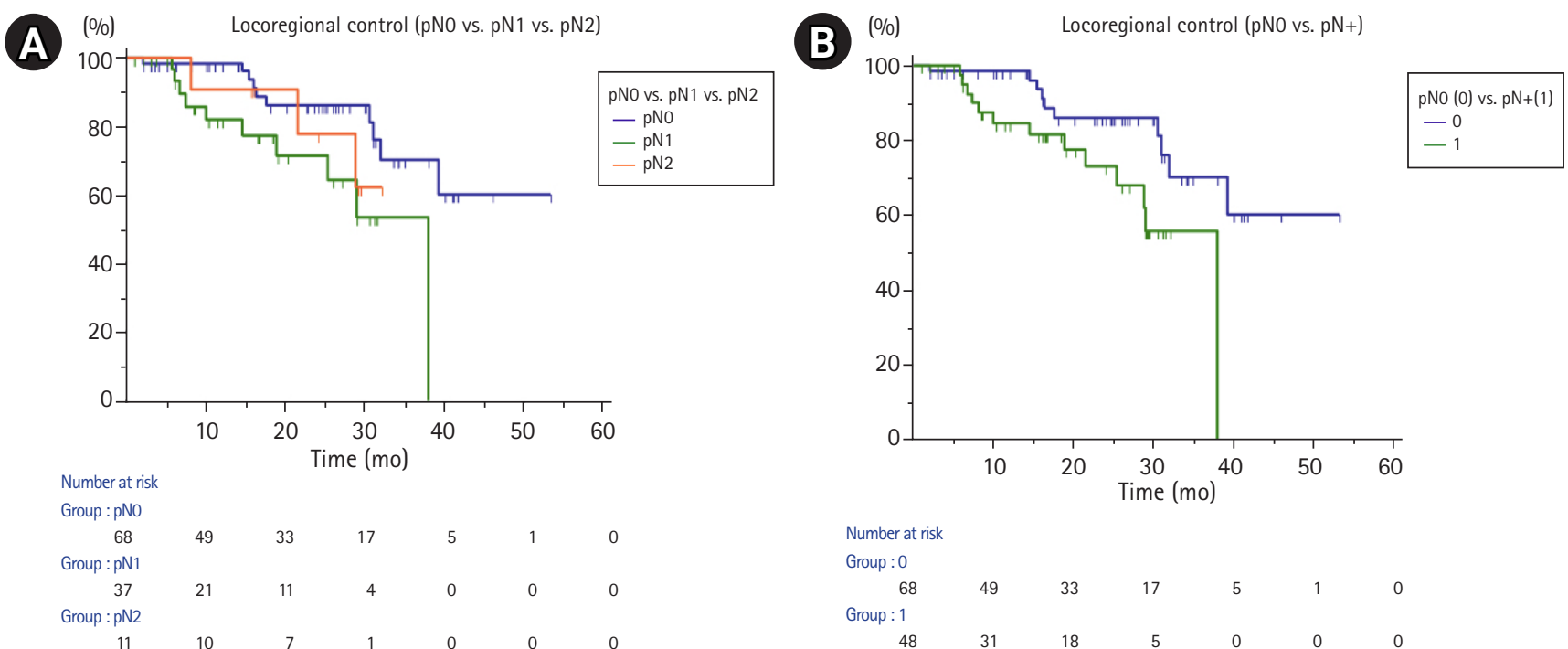

Fig. 2. Kaplan-Meier curves depicting the variation of locoregional control with (A) pN (0 vs. 1 vs. 2) and (B) pN (0 vs. 1/2). 
events in T1, T2, T3, and T4 were 4/11, 14/63, 3/20, and 4/22, respectively, for calculation of OS. On performing an univariate analysis, tumor stage, pathological tumor size, LVI, PNI and DOI didn't impact the OS (Table 2). However, statistically significant results were noticed with pathological nodal staging. There was statistically significant improved outcomes with pathological node negativity. Presence of pathologically positive nodes was significantly inversely correlated with OS; (pNO vs. pN1+2: $86.6 \%$ vs. $68.6 \%$; $p$ $=0.015$; Fig. $3 \mathrm{~A}$ ) and (pN0 vs. pN1 vs. pN2: $86.6 \%$ vs. $71.7 \%$ vs. $64.9 \% ; p=0.045)$, respectively.

\section{Disease-free survival}

The 2-year DFS rate was 77.4\%. The percentage of events in pN0, pN1, and pN2 cases for calculation of DFS were 11/68 (16\%), 11/37 (30\%), and 4/11(36\%), respectively. Among the prognostic factors, only pathological $\mathrm{N}$ staging was associated statistically significant decrease in 2-year DFS rates with nodal positivity; (pN0 vs. pN+: $86.5 \%$ vs. $74.9 \%$; $p=0.010$; Fig. $3 \mathrm{~B}$ ) and (pN0 vs. $p N 1$ vs. $p N 2$ : $84.3 \%$ vs. $69.6 \%$ vs. $64.9 \% ; p=0.032$ ), respectively.

\section{Failure patterns}

Twenty-three patients had locoregional failures. Of these $30.4 \%$ (7/23) cases failed within 12 months, while 60.8\% (14/23) patients failed within 2 years. Eleven cases had isolated local failure without regional failure, while 12 had isolated regional failure. Four patients had contralateral neck failures. Five patients had isolated distant failures. The crude contralateral lymph node failure (CLNF) rate and 2-year contralateral neck recurrence control rate were $3.4 \%(4 / 116)$ and $94.9 \%$, respectively. The most signifi- cant factor affecting CLNF was nodal status. Statistically significant difference in CLNF rates were found with higher nodal burden (pNo vs. pN1 vs. pN2: 100\% vs. $97.1 \%$ vs. 71.4\%; $p=0.001$ ) and positive nodal status (pN0 vs. pN1+2: $100 \%$ vs. $86.3 \%$; $p=$ 0.009). On further analysis, it was found that 3 of 4 patients having contralateral neck failures had $\mathrm{N} 2 \mathrm{~b}$ disease. One patient with contralateral neck failure had N1 disease leading to a crude failure rate of $2.7 \%$ (1/37 patients). The histopathological feature of patients with contralateral neck failure were as follows: patient \#1 (pT2N2b, PNI+, LVI+, moderately differentiated, clear margins, DOI $10 \mathrm{~mm}$ ), patient \#2 (pT4N1, PNI+, moderately differentiated, clear margins, DOI $6 \mathrm{~mm}$ ), patient \#3 (pT2N2b, moderately differentiated, clear margins, DOI $12 \mathrm{~mm}$ ), and patient \#4 (pT1N2b, well differentiated, clear margins, DOI $10 \mathrm{~mm}$ ). All 4 patients had undergone a modified neck dissection. Statistically significant difference in ipsilateral nodal failure rates were found with higher nodal burden (pN0 vs. pN1 vs. pN2: $94.8 \%$ vs. $77.3 \%$ vs. $80.1 \%$; $p=0.033$ ) and positive nodal status ( $\mathrm{pN} 0$ vs. $\mathrm{pN} 1+2: 94.8 \%$ vs. $78.8 \% ; \mathrm{p}=$ 0.038). The crude distant failure rate was $4.3 \%$ (5/116). The 2 -year DMFS was 95.4\%.

\section{Multivariate analysis}

The prognostic factor significantly associated with poorer control rates, that is $\mathrm{pN}$ stage was further evaluated by Cox regression multivariate analysis. In the analysis of pNO vs. $\mathrm{pN}+$, p-value was found significant for OS (hazard ratio [HR] $=0.372$; 95\% confidence interval $[\mathrm{Cl}], 0.16-0.85 ; \mathrm{p}=0.02)$ and DFS $(H R=0.356$; $95 \% \mathrm{Cl}, 0.15-0.81 ; p=0.04)$. Nodal stage N2 was associated with worst outcomes for OS, DFS, and LRC.

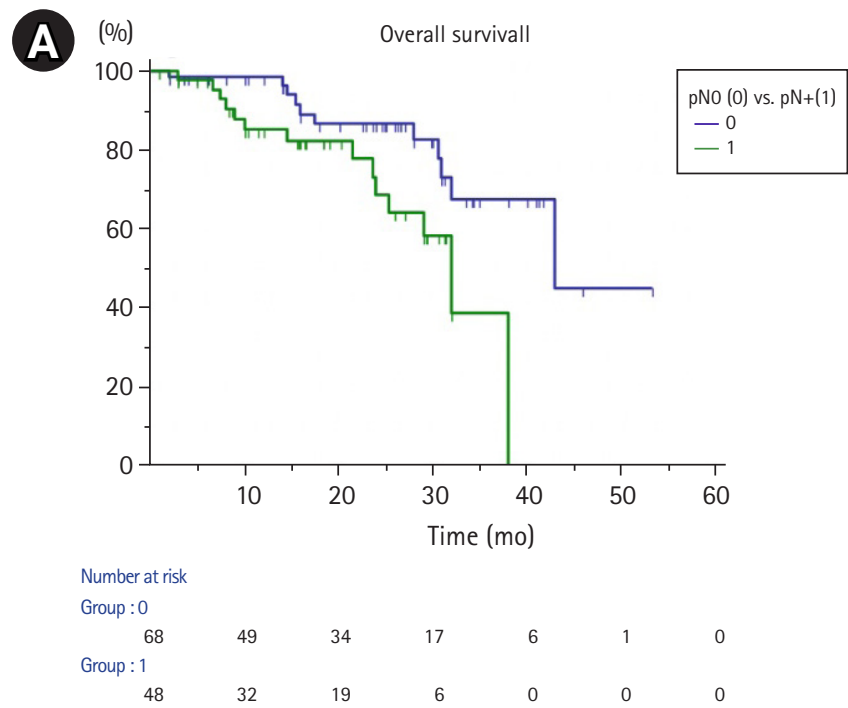

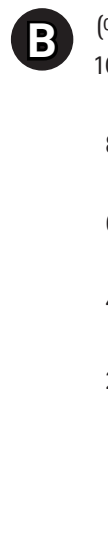
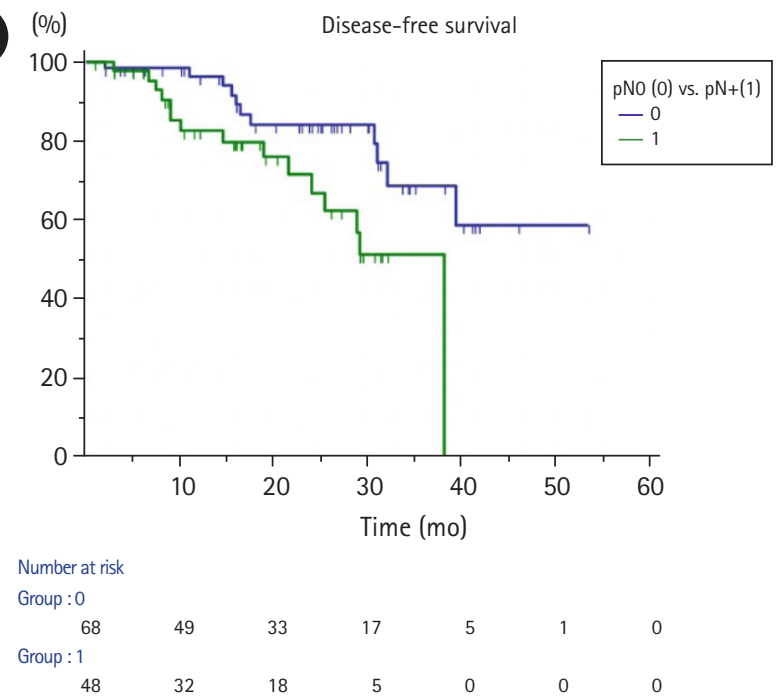

Fig. 3. Kaplan-Meier curves depicting (A) overall survival and (B) disease-free survival variation with nodal status. 


\section{Discussion and Conclusion}

This retrospective review aims to analyse prognostic factors and outcomes associated with the treatment of buccal mucosa cancers receiving ipsilateral radiotherapy in a tertiary cancer centre. Ghoshal et al. [16] reported $80 \%$ oral cancers presenting with stage III and IV. Our cohort had similar findings at $67 \%$ advanced cancers at presentation. The decision of need for ipsilateral or bilateral neck radiotherapy is generally arrived upon after assessing the clinicopathological features in the post-operative histopathology and the risk of nodal drainage to ipsilateral and contralateral lymph nodes [8]. The risk of nodal metastasis leading to neck failure varies among different sites of oral cavity in the range of 30\%$40 \%$ [17]. For buccal mucosa, the risk ranges from 10\% to 30\% [18-20], while for other sites like tongue it is generally mentioned in the range of 15\%-75\% [21-24]. Buccal cancers have higher neck control rates as compared to sites like tongue, as reported by Liao et al. [24], 5-year neck control rate of 93\% versus $86 \%$ ( $p=$ $0.0115)$ in a retrospective comparison of buccal and tongue cancers.

Several authors have previously reported on unilateral radiation therapy in well-lateralized oral cavity and oropharyngeal cancers $[10,13,18,25]$ with excellent outcomes in terms of survival and contralateral neck control, but limited reports have been published addressing outcomes in cases of buccal cancers treated with unilateral irradiation. Publications by Vergeer et al. [13] and Cerezo et al. 18] have reported 5-year OS rates of $61 \%$ and $82.5 \%$, respectively, in a combined cohort of oral cavity and oropharyngeal malignancies. Both these studies included a mixed bag of patients, and hence the outcomes cannot be directly applied to a site like buccal mucosa, which is generally well lateralized. One of the larger studies focusing on outcomes of combined modality treatment in buccal mucosa cancers was reported by Lin et al. [8], who reported outcomes of 145 cases, of which 125 were treated with ipsilateral irradiation. The 5-year OS was 55\%. However, it included all types of buccal mucosa cancers, including those with extracapsular extension which accounted for $31.7 \%$ patients warranting concurrent chemoradiotherapy. So, the inclusion of high risk patients may have resulted in inferior outcomes compared to the cohort of patients in the previously mentioned studies. Another report by Habib et al. [26] addresses the results of unilateral face and neck treatment in 481 oral cavity cancers, but only 165 cases received combined modality treatment. The current report is one of the first study addressing the outcomes of unilateral irradiation in buccal mucosa cancers.

The incidence of various prognostic factors was comparable to that of other studies, PNI was present in 26.7\% [27], while moder- ately differentiated tumors were around $48 \%$. DeConde et al. [27] have reported 29\% incidence of PNI and moderately differentiated tumors were 48\%. Diaz et al. [22] and Pop et al. [28] have reported the timing of locoregional failure to be 12 months or before 24 months. While most of the recurrences tend to happen within the first 2 years [16], the incidence of recurrence in neck varies from 5\%-10\% for early cancers [13] to $25 \%-30 \%$ in advanced cases [22]. Our outcomes also corroborate with the literature with most failures (63.6\%) happening within 24 months. The current study failed to show a predictive relationship between $\mathrm{pT}$ stage and overall TNM stage. This could be attributed possibly to the fact that, the patients with early stage tumors, i.e., pT1/2 tumors were associated with more number of other poor prognostic factors and also that is the reason they received radiotherapy. Nodal staging was associated with significantly poor outcomes in terms of OS, DFS, and LRC. Pathological node positive $(\mathrm{pN}+)$ disease status was associated with statistically significant differences in OS, DFS, LRC, and RC, although pN+ status didn't influence LC in our study. No significant differences were found in OS with LVI, PNI or grade, although there was a trend towards significant decrement in OS with $\mathrm{LVI}+$ disease and grade.

There is paucity of literature addressing CLNF following unilateral neck surgery and irradiation in buccal cancers. The incidence of CLNF varies according to the site of primary tumor. For oral cavity cancers, it is reported to be $0.9 \%-36 \%$ [29-32]. However, for anatomical site like buccal mucosa which has drainage largely to ipsilateral lymph nodal regions, have negligible rates of contralateral lymph node recurrence ranging from 0\%-5.7\% (Table 3). The chances of CLNF increase with T stage [29-31]. However, some other studies suggest no variation in CLNF with T stage [13,32]. Several other studies mention variation with grade of the tumor $[26,31,33,34]$ while one study by Kowalski et al. [28] suggests no association of CLNF with differentiation status. Presence of nodes draining ipsilaterally entail a poor prognosis and increase the risk of CLNF [13,26,32-36]. Twenty-seven percent (13/48) cases with $\mathrm{pN}+$ disease had locoregional failures in our study. Four out of 48 $\mathrm{pN}+$ cases (8.3\%) had CLNF in our cohort. Capote-Moreno et al. [33] have reported similar outcomes with 21.6\% patients showing contralateral neck failures in patients presenting with $\mathrm{pN}+$ disease and 6.4\% contralateral neck failure rates in ipsilateral nodal disease. Table 3 shows studies assessing various prognostic factors which affect the rate of involvement of contralateral lymph node in buccal mucosa cancers.

Ipsilateral radiotherapy decreases doses to contralateral salivary gland, thereby decreasing incidence of xerostomia [35]. The ipsilateral fields also cause decrease rates of mucositis as lower volumes of oral mucosa is irradiated [36]. There is growing evidence in the 
Table 3. Studies depicting CLNF in buccal mucosa cancers and factors affecting it

\begin{tabular}{|c|c|c|c|c|c|c|}
\hline Study & Year & OC cases & BM & Cohort & Incidence of CLNF & Factors increasing incidence of CLNF \\
\hline Lin et al. [8] & 2008 & 142 & All & BM only & $3 / 142(2.1)$ & - \\
\hline $\begin{array}{l}\text { Gonzalez-Garcia } \\
\text { et al. [34] }\end{array}$ & 2008 & 315 & All & BM only & 18/315 (5.7) & $\begin{array}{l}\text { Time to diagnosis ( } p=0.03) \text {, clinical TNM tumor stag- } \\
\text { ing }(p=0.03) \text {, ipsilateral clinical N status }(p=0.04) \text {, } \\
\text { grade }(p=0.04), \text { margins }(p=0.007) \text {, type of neck } \\
\text { dissection }(p=0.04) \text {, PNI }(p=0.0002)\end{array}$ \\
\hline $\begin{array}{l}\text { Capote-Moreno } \\
\text { et al. [33] }\end{array}$ & 2010 & $\begin{array}{c}\mathrm{OC}+\mathrm{OPX} \\
402\end{array}$ & 31 & $\mathrm{OC}+\mathrm{OPX}$ & $\begin{array}{c}23 / 296 \text { (7.7) for } \mathrm{OC} \\
1 / 31 \text { (3.2) for } \mathrm{BM}\end{array}$ & $\begin{array}{l}\text { Gender, tumor location, homolateral positive nodes, } \\
\text { tumor extension across the midline, histologic grade, } \\
\text { margin status, pattern of growth, and perineural } \\
\text { spread }\end{array}$ \\
\hline Fang et al. [23] & 2013 & 67 & All & BM only & $0 / 67(0.0)$ & Poor differentiation, regional lymph node metastasis \\
\hline Habib et al. [26] & 2015 & 481 & 46 & $\mathrm{OC}$ & $\begin{array}{l}\text { 14/481 (2.9), } 2 / 46 \\
\text { (4.34) for BM }\end{array}$ & $\begin{array}{l}\text { Poor differentiation }(p=0.037) \text {, ipsilateral nodal me- } \\
\text { tastasis }(p=0.006)\end{array}$ \\
\hline Present study & & 116 & All & BM only & $4 / 116(3.4)$ & Multiple ipsilateral lymph node metastasis (N2b) \\
\hline
\end{tabular}

Values are presented as number (\%).

OC, oral cavity; BM, buccal mucosa; CLNF, contralateral lymph node failure; OPX, oropharynx; LVI, lymphovascular invasion; PNI, perineural invasion.

literature that ipsilateral radiotherapy in oral cavity cancers is as good as bilateral radiotherapy, with better side effect profile [37]. The effectiveness of ipsilateral radiotherapy in a particular group of patients is because of low incidence of contralateral nodal involvement. In the previously published series by Lin et al. [8] which evaluated treatment outcomes in buccal mucosa cancers, it was opined by the authors that bilateral irradiation was given more to patients with N2 disease, but it conferred no significant benefit ( $p=0.95$ ) over unilateral radiotherapy in terms of LRC. Based on these facts, they recommend unilateral radiotherapy in cases with multiple lymph nodes.

Based on our results, we recommend that contralateral neck irradiation may be avoided safely in intermediate risk group of patients as per inclusion criteria of our study. We also recommend individualizing the balance between the risk of contralateral nodal failure and expected toxicity of bilateral irradiation while considering ipsilateral or bilateral irradiation in buccal mucosa cancers particularly in patients with multiple number or level of ipsilateral nodal involvement.

Certain limitations associated with this analysis have been enumerated. Firstly, the report is retrospective in nature. Secondly, we have reported the study with a median follow-up of 2 years, although it is worth considering that most of the failures happen within the first 2 years [16]. The above-mentioned limitations, however, highlight the importance of publishing single institutional data, which are eventually consolidated through meta-analysis. Moreover, this type of data is likely to come from retrospective studies only, thereby further highlighting the need to address this issue, so that we can exclude that cohort of patients from ipsilateral radiation which have high likelihood of failure, either locally or regionally, including ipsilateral and contralateral failures. Strength of the study includes a pure patient sample with homogenous uni- form treatment, and to the best of our knowledge and as per the literature review the first such study addressing a ipsilateral radiotherapy in buccal mucosa cancers. This study will further add to the understanding of the pattern of failures and addresses the cohort of patients which might benefit the most by irradiation of the bilateral rather ipsilateral neck.

In conclusion, the incidence of contralateral recurrence in buccal mucosa cancer patients treated with primary surgery and adjuvant ipsilateral irradiation is low. It points to the fact that buccal mucosa carcinoma patients with intermediate risk factors can be spared of bilateral irradiation, without significantly affecting the OS and $\mathrm{LRC}$ rates. Patients with multiple pathologically involved nodes are at higher risk for developing contralateral regional recurrence as compared to node-negative disease.

\section{Conflict of Interest}

No potential conflict of interest relevant to this article was reported.

\section{References}

1. Bray F, Ferlay J, Soerjomataram I, Siegel RL, Torre LA, Jemal A. Global cancer statistics 2018: GLOBOCAN estimates of incidence and mortality worldwide for 36 cancers in 185 countries. CA Cancer J Clin 2018;68:394-424.

2. Elango JK, Gangadharan P, Sumithra S, Kuriakose MA. Trends of head and neck cancers in urban and rural India. Asian Pac J Cancer Prev 2006;7:108-12

3. Iyer SG, Pradhan SA, Pai PS, Patil S. Surgical treatment outcomes of localized squamous carcinoma of buccal mucosa. Head Neck 2004;26:897-902. 
4. Bernier J, Domenge $C$, Ozsahin $M$, et al. Postoperative irradiation with or without concomitant chemotherapy for locally advanced head and neck cancer. N Engl J Med 2004;350:1945-52.

5. Huang SH, Hwang D, Lockwood G, Goldstein DP, O'Sullivan B. Predictive value of tumor thickness for cervical lymph-node involvement in squamous cell carcinoma of the oral cavity: a meta-analysis of reported studies. Cancer 2009;115:1489-97.

6. Indian Council of Medical Research. Guidelines for management of buccal mucosa cancer. New Delhi, India: Indian Council of Medical Research; 2010.

7. Cooper JS, Pajak TF, Forastiere AA, et al. Postoperative concurrent radiotherapy and chemotherapy for high-risk squamous-cell carcinoma of the head and neck. N Engl J Med 2004;350:1937-44.

8. Lin CY, Lee LY, Huang SF, et al. Treatment outcome of combined modalities for buccal cancers: unilateral or bilateral neck radiation? Int J Radiat Oncol Biol Phys 2008;70:1373-81.

9. Liao CT, Wang HM, Ng SH, et al. Good tumor control and survivals of squamous cell carcinoma of buccal mucosa treated with radical surgery with or without neck dissection in Taiwan. Oral Oncol 2006;42:800-9.

10. Liu H, Tam L, Woody NM, et al. Risk of contralateral nodal failure in well lateralized early T-stage oral cavity cancer receiving unilateral treatment. Int J Radiat Oncol Biol Phys 2018;102: S22-S23.

11. Gregoire V, Coche E, Cosnard G, Hamoir M, Reychler H. Selection and delineation of lymph node target volumes in head and neck conformal radiotherapy: proposal for standardizing terminology and procedure based on the surgical experience. Radiother Oncol 2000;56:135-50.

12. Lanzer M, Zemann W, Lübbers $T$, Kruse $A$, Reinisch $S$. Do patients with oral and oropharyngeal squamous cell carcinoma benefit from elective contralateral neck dissection? A long-term analysis. Head Neck Oncol 2012;4:70.

13. Vergeer MR, Doornaert PA, Jonkman A, et al. Ipsilateral irradiation for oral and oropharyngeal carcinoma treated with primary surgery and postoperative radiotherapy. Int J Radiat Oncol Biol Phys 2010;78:682-8.

14. Langendijk JA, Slotman BJ, van der Waal I, Doornaert P, Berkof J, Leemans CR. Risk-group definition by recursive partitioning analysis of patients with squamous cell head and neck carcinoma treated with surgery and postoperative radiotherapy. Cancer 2005;104:1408-17.

15. Edge SB, Byrd DR, Compton CC, Fritz AG, Greene FL, Trotti A. AJCC cancer staging manual. 7th ed. New York, NY: Springer; 2010.

16. Ghoshal S, Mallick I, Panda N, Sharma SC. Carcinoma of the buccal mucosa: analysis of clinical presentation, outcome and prog- nostic factors. Oral Oncol 2006;42:533-9.

17. Chen AY, Myers JN. Cancer of the oral cavity. Dis Mon 2001;47: 275-361.

18. Cerezo L, Martin M, Lopez M, Marin A, Gomez A. Ipsilateral irradiation for well lateralized carcinomas of the oral cavity and oropharynx: results on tumor control and xerostomia. Radiat Oncol 2009;4:33.

19. Myers E. Cancer of the head and neck. 4th ed. Philadelphia, PA: Saunders; 2003.

20. Strong EW. Carcinoma of the tongue. Otolaryngol Clin North Am 1979;12:107-14.

21. Lindberg R. Distribution of cervical lymph node metastases from squamous cell carcinoma of the upper respiratory and digestive tracts. Cancer 1972;29:1446-9.

22. Diaz EM Jr, Holsinger FC, Zuniga ER, Roberts DB, Sorensen DM. Squamous cell carcinoma of the buccal mucosa: one institution's experience with 119 previously untreated patients. Head Neck $2003 ; 25: 267-73$.

23. Fang FM, Leung SW, Huang $C_{\text {, }}$ et al. Combined-modality therapy for squamous carcinoma of the buccal mucosa: treatment results and prognostic factors. Head Neck 1997;19:506-12.

24. Liao CT, Huang SF, Chen IH, et al. Tongue and buccal mucosa carcinoma: is there a difference in outcome? Ann Surg Oncol 2010;17:2984-91.

25. O'Sullivan B, Warde P, Grice B, et al. The benefits and pitfalls of ipsilateral radiotherapy in carcinoma of the tonsillar region. Int J Radiat Oncol Biol Phys 2001;51:332-43.

26. Habib M, Murgasen J, Gao K, et al. Contralateral neck failure in lateralized oral squamous cell carcinoma. ANZ J Surg 2016;86:188-92.

27. DeConde A, Miller ME, Palla B, Lai C, Elashoff D, Chhetri D, St John MA. Squamous cell carcinoma of buccal mucosa: a 40-year review. Am J Otolaryngol 2012;33:673-7.

28. Pop LA, Eijkenboom WM, de Boer MF, et al. Evaluation of treatment results of squamous cell carcinoma of the buccal mucosa. Int J Radiat Oncol Biol Phys 1989;16:483-7.

29. Koo BS, Lim YC, Lee JS, Choi EC. Management of contralateral NO neck in oral cavity squamous cell carcinoma. Head Neck 2006;28:896-901.

30. Kowalski LP, Bagietto R, Lara JR, Santos RL, Tagawa EK, Santos IR. Factors influencing contralateral lymph node metastasis from oral carcinoma. Head Neck 1999;21:104-10.

31. Kurita $H$, Koike $T$, Narikawa JN, et al. Clinical predictors for contralateral neck lymph node metastasis from unilateral squamous cell carcinoma in the oral cavity. Oral Oncol 2004;40:898-903.

32. Martin $H$, Del Valle $B$, Ehrlich $H$, Cahan WG. Neck dissection. Cancer 1951:4:441-99. 
33. Capote-Moreno A, Naval L, Munoz-Guerra MF, Sastre J, Rodriguez-Campo FJ. Prognostic factors influencing contralateral neck lymph node metastases in oral and oropharyngeal carcinoma. J Oral Maxillofac Surg 2010;68:268-75.

34. Gonzalez-Garcia R, Naval-Gias L, Sastre-Perez J, et al. Contralateral lymph neck node metastasis of primary squamous cell carcinoma of the tongue: a retrospective analytic study of 203 patients. Int J Oral Maxillofac Surg 2007;36:507-13.

35. Eisbruch A, Kim HM, Terrell JE, Marsh LH, Dawson LA, Ship JA. Xerostomia and its predictors following parotid-sparing irradia- tion of head-and-neck cancer. Int J Radiat Oncol Biol Phys 2001;50:695-704.

36. Jensen $A B$, Hansen 0 , Jorgensen $K$, Bastholt $L$. Influence of late side-effects upon daily life after radiotherapy for laryngeal and pharyngeal cancer. Acta Oncol 1994;33:487-91.

37. Corvo R, Foppiano F, Bacigalupo A, Berretta L, Benasso M, Vitale V. Contralateral parotid-sparing radiotherapy in patients with unilateral squamous cell carcinoma of the head and neck: technical methodology and preliminary results. Tumori 2004;90:66-72. 